\title{
Correlation between sperm ultrastructure in infertile patients with abnormal sperm morphology and DNA damage
}

\author{
M. He and L. Tan \\ The Second Affiliated Hospital of Zhengzhou University, Henan, China \\ Corresponding author: L. Tan \\ E-mail: xyrhbkj@163.com \\ Genet. Mol. Res. 14 (4): 17000-17006 (2015) \\ Received August 18, 2015 \\ Accepted December 1, 2015 \\ Published December 15, 2015 \\ DOI http://dx.doi.org/10.4238/2015.December.15.6
}

\begin{abstract}
This study explored the correlation between sperm ultrastructure in infertile patients with abnormal sperm morphology and DNA damage. Three unusual sperm morphologies were selected for the experimental group namely case 1 (95\% headless sperm), case $2(98 \%$ headless sperm), and case $3(100 \%$ headless sperm), and the control group consisted of 2 subjects ( 20 and $15 \%$ headless sperm). For case 1 , the patient was negative for sexually transmitted diseases and had normal semen plasma biochemistry, reproductive hormones, peripheral blood chromosomes, and azoospermia factor (AZF). The aneuploid rate of sperm chromosomes was $0.6 \%$, and DNA damage index of sperm nuclei was $84.4 \%$. The partner of this patient did not get pregnant after artificial reproductive technology assistance. For case 2 , the aneuploid rate of sperm chromosomes was $0.8 \%$ and DNA damage index of sperm nuclei was $95 \%$. This patient and his spouse did not choose assisted reproduction. For case 3 , reproductive hormones, peripheral blood chromosomes and AZF were normal and the aneuploid rate of sperm chromosomes was $0.2 \%$. The wife of this patient gave birth to a healthy baby after ova removal, fertilization and transplantation. For the control group, the aneuploid rate of sperm chromosomes and DNA damage index of sperm nuclei were approximately
\end{abstract}


0.3 and $30 \%$, respectively. To sum up, sperm ultrastructure of infertile patients suffering from unusual sperm morphology is associated with DNA damage to some extent and can cause infertility. However, pregnancy is still possible through intracytoplasmic sperm injection.

Key words: Sperm morphology; Sperm ultrastructure; DNA damage; Teratozoospermia

\section{INTRODUCTION}

Male infertility is defined as the inability of a male to induce pregnancy in a fertile female (Ramlingam et al., 2014; Yuen et al., 2014). Traditional routine examinations examine the number, density, aberration rate, and activity ratio of sperm, and the levels of anti-sperm antibodies in infertile male patients. Recent studies have found that these parameters in some patients with idiopathic infertility are also affected by other factors, including DNA integrity (Bezold et al., 2007). Patients with reduced, weak or abnormal sperm are expected to be able to bear their own biological children by using assisted reproductive technologies (ART), with intracytoplasmic sperm injection (ICSI) being particularly successful (VandenMeerschaut et al., 2014). Sperm with genetic defects can still be directly injected into an ovum through ICSI as the sperm does not go through natural selection. Therefore, the influence of DNA integrity of sperm on reproduction is drawing more attention (Marchesi et al., 2010; Zribi et al., 2012).

Globozoospermia with detects in the head of sperm is rarely seen, with the incidence of $<0.1 \%$ in male infertile patients. Under a light microscope, the head of sperm is observed to be round, with abnormal or missing acrosome and accompanied with messy middle piece and tail. Round head of sperm, no acrosome, a layer of uniform unit membrane around the nucleus and disorders in the arrangement of mitochondria and microtubule in tail are also visible under an electronic microscope. The ability of sperm movement is an important index measured in male fertility clinics and is of great significance for fertilization (Ren et al., 2001). Only active sperm are capable of fertilization via the genital tract, and the entire insemination process requires sperm to pass through the cumulus oophorus and zona pellucida (ZP). Abnormal sperm morphology, known as teratozoospermia, seriously affects this process. This study analyzed the relationship between the ultrastructure of abnormal sperm and DNA damage, which contributes to exploring fertility mechanisms of patients with abnormal sperm morphology and provides the basis for clinical treatment.

\section{MATERIAL AND METHODS}

\section{Research subjects}

Three unusual sperm morphologies were selected from three males: case 1 was 42 years old and had $95 \%$ headless sperm; case 2, 24 years old, 98\% headless sperm; and case 3,28 years old, $100 \%$ headless sperm. All cases had typical sexual libido but had been infertile for 11, 1 and 5 years, respectively, without the use of birth control. The control group included two cases, with 20 and $15 \%$ headless sperm. This experiment was approved by the ethics committee of The Second Affiliated Hospital of Zhengzhou University (China) and all patients provided written informed consent. 


\section{Inclusion and exclusion criteria}

Healthy males (mean age: $34.5 \pm 2.9$ years) had normal sex lives after marriage, but were infertile for over 1 year without any birth control. Routine medical examinations and semen tests were deemed normal of all included patients (volume $\geq 2 \mathrm{~mL}, \mathrm{pH} \geq 7.2$; density $\geq 20 \times 10^{6}$ / $\mathrm{mL}$; percentage of sperm forward movement $\geq 50 \%$ ). Males were excluded if they experienced trauma or sexual dysfunction, had a family history of hereditary disease, or showed obvious signs of testicular, epididymis or ductus deferens abnormities.

\section{Information collection, blood sample acquisition, and follow-up}

Patients' clinical information was collected by preoperative examination and questionnaire, including age, sex, profession, childbearing history, family history, reproductive age of their parents, and existence of consanguineous marriage. Peripheral blood (3-4 mL) was drawn from patients and treated with heparin for peripheral blood chromosome $\mathrm{G}$ banding karyotype analysis. Another $2 \mathrm{~mL}$ blood was drawn and treated with the anticoagulant ethylene diamine tetraacetic acid (EDTA) for Y-chromosome micro missing analysis. Moreover, $5 \mathrm{~mL}$ peripheral blood was drawn from both partners and used for reproductive hormone detection. All patients were required to sign informed consent before ART treatment and cooperate with clinical pregnancy outcome observation and telephone follow-up on other relevant issues.

\section{Analysis of semen and sperm morphology}

Semen was collected from males following sexual abstinence for 2-7 days, after which it was stored in an aseptic container, liquefied in a water bath at $37^{\circ} \mathrm{C}$, and subsequently examined. Dynamic parameters of semen were detected using the WeiLi color sperm quality analysis system (WLJY-9000, WeiLi, China) and analyzed according to parameters established by the World Health Organization (WHO). Sperm morphology smears were performed by published methods (Menkveld et al., 1996). Briefly, the acrosome form of sperm was analyzed and the acrosome index and integrity rate were calculated.

\section{Ovulation induction treatment and insemination}

The female partners of all cases were treated with a subcutaneous injection of gonadotropinreleasing hormone agonist ( $\mathrm{GnRHa}$, Merck Serono, Germany) for ovulation induction continuously for 10-16 days until reaching the standard [follicle-stimulating hormone (FSH) $<5 \mathrm{mlU} /$ $\mathrm{mL}$; luteinizing hormone $(\mathrm{LH})<5 \mathrm{mlU} / \mathrm{mL}$; estradiol (E2) $<50 \mathrm{pg} / \mathrm{mL}$; antral follicle count (AFC) $<5$ mm]. Recombinant FSH (r-FSH, Merck Serono, Germany) and urinary FSH (u-FSH, Lishenbao, Lizhu Pharmaceutical, China) were injected subcutaneously to promote ovulation, and human menopausal gonadotropin (HMG, Lizhu Pharmaceutical) was injected subcutaneously in the ovulatory period. Human chorionic gonadotropin (HCG, Lizhu Pharmaceutical) was injected intramuscularly when the diameter of the dominant follicle was $\geq 18 \mathrm{~mm}$ and the ovum was collected $34-36 \mathrm{~h}$ later.

Density gradient centrifugation combined with the swim-up method (Xu et al., 2010) was applied in processing the liquefied semen, resulting in asemen volume of $600 \mu \mathrm{L}$ and concentration of 30,000-50,000 sperm/ovum. Granular cells were eliminated with a mechanical method $4 \mathrm{~h}$ after adding sperm to observe early fertilization conditions, and an early fertilization was confirmed if a bipolar body was found. 


\section{Embryo transplantation and gestation detection}

The embryo was transplanted in to the uterine cavity three days after collection of the ovum and supported using regular progesterone delivery after transplantation. On the fourteenth day after transplantation, HCG levels were tested to determine if there was a biochemical pregnancy (HCG value > $5.3 \mathrm{mlU} / \mathrm{mL}$ was considered pregnant). On the thirty-fifth day, patients who were observed to have a gestational sac via B ultrasound were considered clinically pregnant.

\section{Analysis of sperm ultrastructure}

Sperm was washed twice with $5 \mathrm{~mL} \mathrm{NaCl}$ solution $(0.785 \mathrm{M})$ and centrifuged for $10 \mathrm{~min}$. The supernatant was removed and $2.5 \%$ glutaraldehyde was added drop-wise. Following this, sperm were fixed for $2 \mathrm{~h}$ at $4^{\circ} \mathrm{C}$, fixed twice with osmic acid, embedded with Epon812 epoxy resin (Hyde Venture Biotech Co., Ltd., Beijing, China), sliced in $70 \mathrm{~nm}$ (under a light microscope), and dyed using toluidine blue. Sperm were observed with a JEM-1200EX (Olympus, Japan) transmission electron microscope.

\section{Statistical methods}

SPSS 17.0 software (Chicago, IL, USA) was used for statistical analysis and data were expressed as mean \pm standard deviation (SD). Before analysis, it was confirmed that data was normally distributed. Data were analyzed using Student $t$-test of two independent samples if normally distributed; otherwise, the rank sum test was used. Numerical data were expressed as a percentage and analyzed using the four-fold table chi-square test. Differences were considered to be statistically significant if $P<0.05$.

\section{RESULTS}

\section{Analysis of headless sperm cases}

Routine semen examination of case 1 (95\% headless sperm) was found to be normal during liquidation, with moderate stickiness, volume $1.5 \mathrm{~mL}, \mathrm{pH} 7.9$, concentration $83.55 \times 10^{6} / \mathrm{mL}$, and sperm motility $4.19 \%$ in $(\mathrm{a}+\mathrm{b})$ level (Figure1A and 1B). Chlamydia, mycoplasma, gonococcus, and toxoplasma gondii + rubella virus + cytomegalovirus + herpes simplex virus (TORCH) were negative, and seminal plasma biochemistry, reproductive hormones, peripheral blood chromosomes and azoospermia factor (AZF) were all normal. The aneuploid rate of sperm chromosomes was $0.6 \%$ and the damage index of sperm nuclei, evaluated by sperm DNA fragmentation index (SDFI), was $84.4 \%$. Ten ova were collected from the wife of case 1 , and four oocytes with good morphology in the meiotic period for the second time (M II stage) were selected and ICSI was performed with active sperm. Following this, 2 pronucleuses formed, and two good-quality embryos were transplanted. However, the wife did not become pregnant after transplantation.

Routine semen examination of case 2 ( $98 \%$ headless sperm) was also found to be normal during the liquidation process and presented with moderate stickiness, volume $3.1 \mathrm{~mL}$, $\mathrm{pH} 7.5$, concentration $26.14 \times 10^{6} / \mathrm{mL}$, $(\mathrm{a}+\mathrm{b})$ level $13 \%$, and survival rate $60 \%$ (Figure $2 \mathrm{~A}$ and $\mathrm{B}$ ). Ureaplasma urealyticum culture was positive and the reproductive hormone prolactin (PRL) was slightly high $(331 \mu \mathrm{lU} / \mathrm{mL})$. Semen anti-sperm antibody (AsAb), peripheral blood chromosome, 
and AZF levels were all normal, and the patient was negative for chlamydia trachomatis (CT). Testis puncture biopsy was performed, involving spermatogonium (20\%), primary spermatocyte $(20 \%)$, secondary spermatocyte $(2 \%)$, spermatid $(25 \%)$, sustentacular cell $(30 \%)$, and sperm $(3 \%)$, and one to two sperms were observed to be fully developed in high power field (HPF) under the microscope. The aneuploid rate of sperm chromosomes was $0.8 \%$ and the DNA damage index of the sperm nuclei was $95 \%$. Different degrees of structural abnormalities were found in the head, neck, middle, and tail of sperm, but the tail could be seen in most sperm (Figure 2A). Acrosome dysplasia and loss, incomplete acrosome membrane structure, nuclear vacuoles (Figure 2B), and uneven and disordered mitochondria (Figure $2 \mathrm{C}$ ) were also observed. Some sperm tails were surrounded with several structurally incomplete axial filaments (Figure 2D). This patient and his partner did not agree to ART treatment.
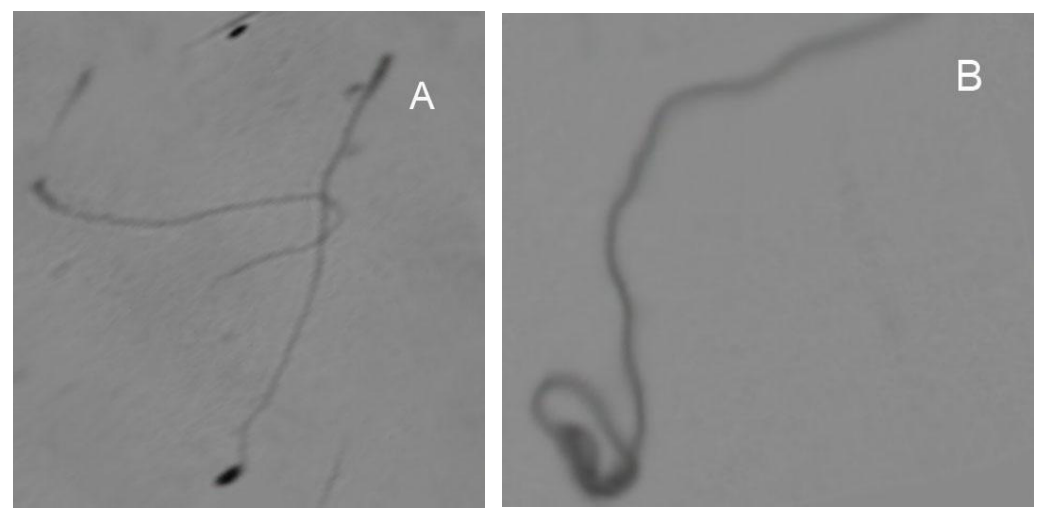

Figure 1. Sperm morphology from case 1 after dyeing with toluidine blue (A: 400X, B: 1000X).
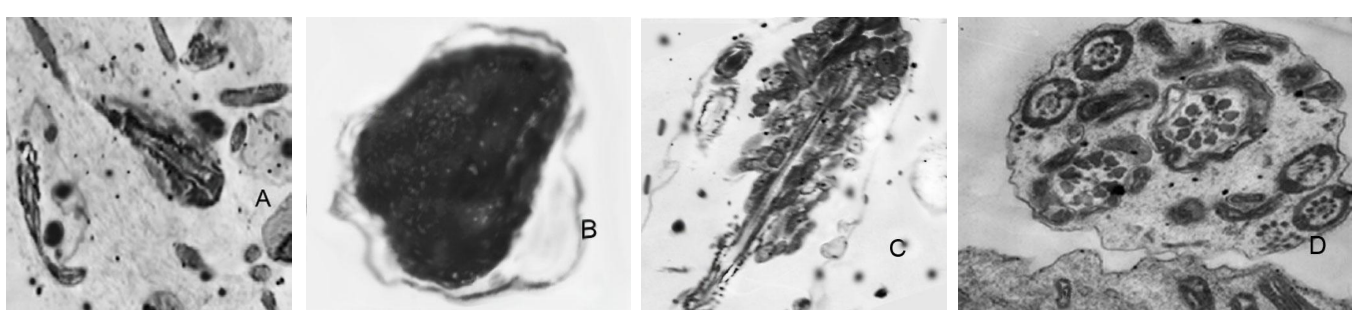

Figure 2. Headless sperm ultrastructure from case 2 as viewed with a transmission electron microscope (TEM). (A) Full sperm (5000X), (B) head (25,000X), (C) middle (12,000X), and (D) cross section of the tail $(25,000 \mathrm{X})$.

In the control group, the aneuploid rate of sperm chromosomes and DNA damage index of sperm nuclei were 0.4 and $34.2 \%$ in the patient with $20 \%$ headless sperm, respectively, and 0.2 and $27.6 \%$ in the patient with $15 \%$ headless sperm, respectively (Table 1 ).

\section{Analysis of globozoospermia cases}

Routine semen examination was performed for case 3 following a 60 min liquidation and showed slight stickiness, $1.2 \mathrm{~mL}$ volume, $\mathrm{pH} 7.8$, concentration $35.99 \times 10^{6} / \mathrm{mL},(\mathrm{a}+\mathrm{b})$ level $0.00 \%$, and survival rate $38 \%$. Fully dyed sperms showed a round head (Figure 3 ). Reproductive 
hormones, peripheral blood chromosomes, and AZF levels were tested and found to be normal, and the aneuploid rate of sperm chromosomes was $0.2 \%$. Twenty-one ova were obtained from this patient's wife, as well as 20 oocytes at MII stage and 1 oocyte at germinal vesicle (GV) stage. ICSI was performed with active sperm. Two PN oocytes were acquired and transplanted to one inferior embryo. After transplantation, the HCG level was $418 \mathrm{U} / \mathrm{L}$ on the 14th day, and a live fetus was observed in utero on the 34th day, accompanied by an embryo and cardiac tube pulse. Finally, a healthy boy was born.

Table 1. DNA integrity and aneuploid rate of chromosomes in sperm of patients with headless sperm.

\begin{tabular}{|c|c|c|c|c|c|c|}
\hline \multirow[t]{2}{*}{ Specimens } & \multirow[t]{2}{*}{ SDFI (\%) } & \multirow{2}{*}{$\begin{array}{c}\text { Aneuploid rate of } \\
\text { sperm chromosomes }\end{array}$} & \multicolumn{2}{|c|}{ Integrated DNA } & \multicolumn{2}{|c|}{ Damaged DNA } \\
\hline & & & No. of haploid & No. of aneuploid & No. of haploid & No. of aneuploid \\
\hline $98 \%$ headless sperm & 95.0 & $0.8 \%$ & 25 & 2 & 472 & 2 \\
\hline $95 \%$ headless sperm & 84.5 & $0.6 \%$ & 76 & 0 & 420 & 3 \\
\hline $20 \%$ headless sperm & 34.2 & $0.4 \%$ & 330 & 1 & 167 & 1 \\
\hline $15 \%$ headless sperm & 27.6 & $0.2 \%$ & 365 & 0 & 135 & 1 \\
\hline
\end{tabular}

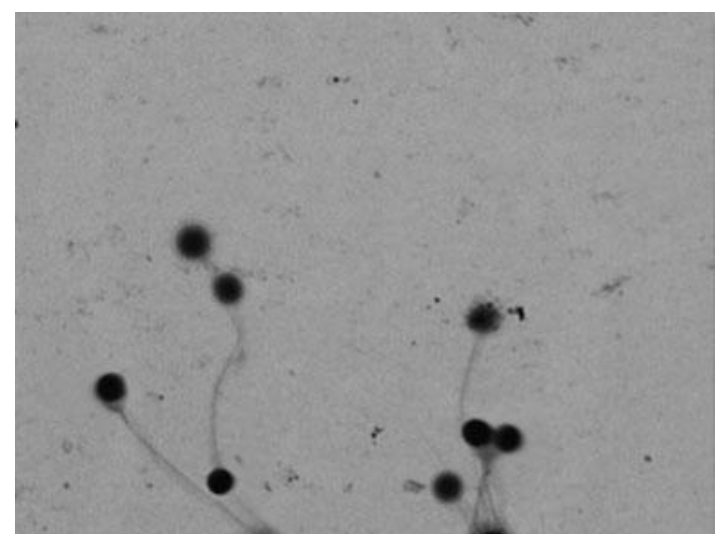

Figure 3. Morphology of globozoospermia from case 3 examined under a light microscope (400X).

\section{DISCUSSION}

Human DNA is of great importance in passing on genetic material and information, and it plays a critical role in human reproduction and genetic characteristics (Slyskova et al., 2014). DNA damage in sperm can be caused by multiple factors, including environmental pollution, diseases, poor lifestyle choices, microelements, and chemical and physical factors (Gong et al., 2014; Wang and Vasquez, 2014). The premise exists that sperm that possess excellent mobility must also have a well-behaved cell nucleus and organelles to provide enough materials and energy. However, DNA damage inside thenucleus will affect the activity of sperm. None of the patients suffering from globozoospermia had any physical issues besides infertility (Chianese et al., 2015). Patients with globozoospermia have no significant differences in sperm concentration, activity or other indexes compared to other infertile patients in routine semen examination; however, the pathogenic mechanism of globozoospermia is not clear. Molecular biology studies (Larson et al., 2001; Brahem et al., 2011) have shown that the formation of round-headed sperm is associated 
with abnormal sperm chromatin condensation and DNA fragmentation. In this study, an inferior single embryo was successfully fertilized in the 3rd day after ICSI, even though the father had globozoospermia, and a healthy baby boy was born, further indicating that ICSI is an effective method for treating globozoospermia. Round-headed sperm hadlittle influence on subsequent developmental potential after insemination, and inferior embryos could be transplanted in the absence of high quality embryos for transplantation.

In conclusion, the sperm ultrastructure of infertile patients with abnormal sperm morphology is correlated with DNA damage to some extent, and a high aberration rate of sperm tends to show a high degree of DNA damage. Considering sperm ultrastructure together with DNA damage, ART should be considered as a fertility treatment for patients with globozoospermia.

\section{Conflicts of interest}

The authors declare no conflict of interest.

\section{ACKNOWLEDGMENTS}

We would like to express my sincere gratitude to all research workers engaged in this study.

\section{REFERENCES}

Bezold G, Politch JA, Kiviat NB, Kuypers JM, et al. (2007). Prevalence of sexually transmissible pathogens in semen from asymptomatic male infertility patients with and without leukocytospermia. Fertil. Steril. 87: 1087-1097.

Brahem S, Mehdi M, Elghezal H and Saad A (2011). Analysis of sperm aneuploidies and DNA fragmentation in patients with globozoospermia or with abnormal acrosomes. Urology 77: 1343-1348.

Chianese C, Fino MG, Riera Escamilla A, López Rodrigo O, et al. (2015). Comprehensive investigation in patients affected by sperm macrocephaly and globozoospermia. Andrology 3: 203-212.

Gong JH, Zhu M, Chu MJ, Sun CQ, et al. (2014). Genetic variants in SMARC genes are associated with DNA damage levels in Chinese Population. Toxicol. Lett. 229: 327-332.

Larson KL, Brannian JD, Singh NP, Burbach JA, et al. (2001). Chromatin structure in globozoospermia: a case report. J. Androl. 22: 424-431.

Marchesi DE, Biederman H, Ferrara S, Hershlag A, et al. (2010). The effect of semen processing on sperm DNA integrity: comparison of two techniques using the novel toluidine blue assay. Eur. J.Obstet. Gynecol. Reprod. Biol. 151: 176-180.

Menkveld R, Rhemrev JP, Franken DR, Vermeiden JP, et al. (1996). Acrosomalmorphology as a novel criterion for male fertility diagnosis: relation with acrosinactivity, morphology (strict criteria), and fertilization in vitro. Fertil. Steril. 65: 637-644.

Ramalingam M, KiniS and Mahmood T (2014). Male fertility and infertility. Obstet. Gynaecol. Reprod. Med. 24: 326-332.

Ren D, Navarro B, Perez G, Jackson AC, et al. (2001). A sperm ion channel required for sperm motility and male fertility. Nature 413: 603-609.

Slyskova J, Lorenzo Y, Karlsen A, Carlsen MH, et al. (2014). Both genetic and dietary factors underlie individual differences in DNA damage Levels and DNA repair capacity. DNA Repair 16: 66-73.

VandenMeerschaut F, Nikiforaki D, Heindryckx B and De Sutter P (2014). Assisted oocyte activation following ICSI fertilization failure. Reprod. Biomed. Online 28: 560-571.

Wang G and Vasquez KM (2014). Impact of alternative DNA structures on DNA damage, DNA repair, and genetic instability. DNA Repair 19: 143-151.

Xu KD, Du YF, Lei YL and Dai RH (2010). A practical method of Ludox density gradient centrifugation combined with protargol staining for extracting and estimating ciliates in marine sediments. Eur. J. Protistol. 46: 263-270.

Yuen RK, Merkoulovitch A, MacDonald JR, Vlasschaert M, et al. (2014). Development of a high resolution Y chromosome microarray for improved male infertility diagnosis. Fertil. Steril. 101: 1079-1085.

Zribi N, Chakroun NF, Ben Abdallah F, Elleuch H, et al. (2012). Effect of freezing-thawing process and quercetin on human sperm survival and DNA integrity. Cryobiology 65: 326-331. 